\title{
Optimal Variational Portfolios with Inflation Protection Strategy and Efficient Frontier of Expected Value of Wealth for a Defined Contributory Pension Scheme
}

\author{
Joshua O. Okoro ${ }^{1}$, Charles I. Nkeki ${ }^{2}$ \\ ${ }^{1}$ Department of Physical Science, Faculty of Science and Engineering, Landmark University, \\ Omu-Aran, Nigeria \\ ${ }^{2}$ Department of Mathematics, Faculty of Physical Sciences, University of Benin, Benin City, Nigeria \\ Email: joshuate@yahoo.com, nkekicharles2003@yahoo.com
}

Received August 16, 2013; revised September 27, 2013; accepted October 26, 2013

Copyright (C) 2013 Joshua O. Okoro, Charles I. Nkeki. This is an open access article distributed under the Creative Commons Attribution License, which permits unrestricted use, distribution, and reproduction in any medium, provided the original work is properly cited.

\begin{abstract}
This paper examines optimal variational Merton portfolios (OVMP) with inflation protection strategy for a defined contribution (DC) Pension scheme. The mean and variance of the expected value of wealth for a pension plan member (PPM) are also considered in this paper. The financial market is composed of a cash account, inflation-linked bond and stock. The effective salary of the plan member is assumed to be stochastic. It was assumed that the growth rate of PPM's salary depends on some macroeconomic factors over time. The present value of PPM's future contribution was obtained. The sensitivity analysis of the present value of the contribution was established. The OVMP processes with inter-temporal hedging terms and inflation protection that offset any shocks to the stochastic salary of a PPM were established. The expected values of PPM's terminal wealth, variance and efficient frontier of the three classes of assets are obtained. The efficient frontier was found to be nonlinear and parabolic in shape. In this paper, we allow the stock price to be correlated to inflation risk index, and the effective salary of the PPM is correlated to inflation and stock risks. This will enable PPMs to determine extents of the stock market returns and risks, which can influence their contributions to the scheme.
\end{abstract}

Keywords: Optimal Variational Merton Portfolios; Mean-Variance; Expected Wealth, Defined Contribution; Pension Scheme; Pension Plan Member; Inter-Temporal Hedging Terms; Stochastic Salary

\section{Introduction}

This paper considers the OVMP strategy under inflation protection, expected wealth and its variance for a DC pension scheme. It was assumed that the salary and risky asset are driven by a standard geometric Brownian motion. The growth rate of salary of PPM is assumed to be a linear function of time. In this paper, we focus on studying the OVMP strategy under inflation protection for a DC pension scheme. In related literature, [1] and [2], studied optimal portfolio strategy based on the non-Gaussian models. They constructed optimal portfolios of variance swaps based on a variance gamma correlated model. The portfolios of the variance swaps are optimized based on the maximization of the distorted expectation given in the index of acceptability. [3] examined the rationale, nature and financial consequences of two alternative ap- proaches to portfolio regulations for the long term institutional investor sectors of life insurance and pension funds. [4] studied the deterministic life styling and gradual switch from equities to bonds in accordance to preset rules. This approach was a popular asset allocation strategy during the accumulation phase of a defined contribution pension plan and was designed to protect the pension funds from a catastrophic fall in the stock market just prior to retirement. They showed that this strategy, although easy to understand and implement, can be highly suboptimal. [5] developed a model for analyzing the ex ante liquidity premium demanded by the holder of an illiquid annuity. The annuity is an insurance product that is similar to a pension savings account with both an accumulation and decumulation phase. They computed the yield needed to compensate for the utility welfare 
loss, which is induced by the inability to re-balance and maintain an optimal portfolio when holding an annuity. [6-8] considered the optimal design of the minimum guarantee in a defined contribution pension fund scheme. They studied the investment in the financial market by assuring that the pension fund optimizes its retribution which is a part of the surplus, which is the difference between the pension fund value and the guarantee. [9] studied optimal investment strategy for a defined contributory pension plan. They adopted dynamic optimization technique. [10] considered optimal portfolio strategies with minimum guarantee and inflation protection for a DC pension scheme. $[11,12]$ studied the optimal portfolio and strategic lifecycle consumption process in a defined contributory pension plan. This work is an extension of [13]. [13] studied the OVMP process of a PPM. In this paper, we introduce additional underlying asset, which is the inflation-linked bond that will protect the investment against inflation risks in the investment profile. [14] considered optimal investment strategy with discounted cash flows. In their work, the investment and the portfolios processes were protected from inflation risks by trading on the index bond asset that is linked to inflation risk over time.

The remainder of this paper is organized as follows. In Section 2, we present the financial market models and wealth process of a PPM. Section 3 presents the expected value of PPM's future contribution, the present value of the expected flow of future discounted contribution, sensitivity analysis of the present value of expected future discounted contribution and value of wealth process of a PPM. In Section 4, we present the optimization process and portfolio value of wealth of a PPM in pension scheme. Also, in this section, some numerical illustrations were made. Section 5 presents the expected wealth and variance of expected wealth for a PPM up to terminal period. Finally, Section 6 concludes the paper.

\section{Problem Formulation}

Let $(\Omega, F, P)$ be a probability space. Let

$$
\wp(F)=\left\{F_{t}: t \in[0, T]\right\},
$$

where

$$
F_{t}=\sigma(W(s): s \leq t)
$$

and the Brownian motion

$$
W(t)=\left(W^{Q}(t), W^{S}(t)\right)^{\prime},
$$

$0 \leq t \leq T$ is a 2-dimensional process, defined on a complete probability space $(\Omega, F, \wp(F), P)$, where $P$ is the real world probability measure and $T$ the terminal time. The vectors $\sigma_{S}(t)$ and $\sigma_{z}(t)$ are the volatility vector for stock and inflation index with respect to changes in $W^{S}(t)$ and $W^{Q}(t)$, respectively. $\mu(t)$ is the appreciation rate for stock. Moreover, $\sigma_{S}(t)$ and $\sigma_{z}(t)$ are the volatilities for the stock and inflation index, respectively, referred to as the coefficients of the market and are progressively measurable with respect to the filtration $F$. We assume that the investor faces a market that is characterized by a risk-free asset (cash account), inflation-linked bond and stock, all of whom are tradeable. In this paper, we allow the stock price to be correlated to inflation risk index and the effective salary of the PPM to be correlated to inflation and stock. This will enables us to check how stock market risks can influence contribution into the scheme.

\subsection{The Financial Model}

The dynamics of the underlying assets are given by (1) and (2)

$$
\begin{gathered}
\mathrm{d} C(t)=r(t) C(t) \mathrm{d} t, C(0)=1 \\
\mathrm{~d} S(t)=S(t)\left(\mu(t) \mathrm{d} t+\sigma_{S}(t) \mathrm{d} W(t)\right), \\
S(0)=s_{0} \\
\mathrm{~d} Z(t, Q(t))=Z(t, Q(t))\left(\left(r(t)+\sigma_{Q}(t) \theta_{z}(t)\right) \mathrm{d} t\right. \\
\left.+\sigma_{z}(t) \mathrm{d} W(t)\right), Z(0)=z,
\end{gathered}
$$

where, $r(t) \in \mathfrak{R}_{+}$is the nominal interest rate, $C(t)$ is the price of the riskless asset at time $t, Z(t, Q(t))$ is the price of the inflation-linked bond at time $t, Q(t)$ is the inflation index at time $t$, and satisfies the dynamics

$$
\mathrm{d} Q(t)=Q(t)\left(E(q(t)) \mathrm{d} t+\sigma_{Q}(t) \mathrm{d} W^{Q}(t)\right),
$$

$E(q(t))$ is the expected rate of inflation which the difference between the nominal interest rate and the real interest rate and $\sigma_{Q}(t)$ is the volatility of inflation index, $S(t)$ is the stock price process at time $t$, $\theta_{\mathrm{z}}(t) \in \mathfrak{R}$ is the inflation price of risk, $\rho \in \mathfrak{R}$ is the correlation coefficient and

$$
\sigma_{S}(t)=\left(\rho \sigma_{S}(t), \sqrt{1-\rho^{2}} \sigma_{S}(t)\right), \quad \sigma_{z}=\left(\sigma_{Q}(t), 0\right) .
$$

The volatility matrice now become

$$
\Sigma^{\prime}(t)=\left(\begin{array}{cc}
\sigma_{Q}(t) & 0 \\
\sigma_{S}(t) \rho & \sigma_{S}(t) \sqrt{1-\rho^{2}}
\end{array}\right)
$$

The market is complete since

$$
\sigma_{Q}(t) \sigma_{S}(t) \sqrt{1-\rho^{2}} \neq 0,
$$

therefore there exists a unique market price of risk vctor, $\theta(t) \in \mathfrak{R}^{2}$ satisfying

$$
\theta(t)=\left(\begin{array}{l}
\theta_{z}(t) \\
\theta_{S}(t)
\end{array}\right)=\left(\begin{array}{c}
\theta_{z}(t) \\
\frac{\mu(t)-r(t)-\rho \sigma_{S}(t) \theta_{z}(t)}{\sigma_{S}(t) \sqrt{1-\rho^{2}}}
\end{array}\right)
$$


The effective salary of the PPM is assume to follow the dynamics

$$
\begin{aligned}
& \mathrm{d} Y(t)=Y(t)\left(\omega(t) \mathrm{d} t+\sigma_{Y}(t) \mathrm{d} W(t)\right), \\
& Y(0)=y_{0}>0,
\end{aligned}
$$

where, $\sigma_{Y}(t)=\left(\sigma_{Y 1}(t), \sigma_{Y 2}(t)\right), \sigma_{Y 1}(t)$ is the volatility caused by the source of inflation, $W^{Q}(t)$ and $\sigma_{Y 2}(t)$ is the volatility caused by the source of uncertainty arises from the stock market, $W^{S}(t)$. We assume that the growth rate of the salary of PPM is linear and satisfies

$$
\omega(t)=a+\beta t, a>0, \beta \geq 0 .
$$

$a$ is seen as expected yearly growth of PPM's salary and $\beta$ economic growth effect or welfare of the PPM. (4) define the buying power of PPM's salary at time $t$.

Suppose that $\sigma_{Y}(t)=(0,0)$, (4) becomes

$$
\mathrm{d} Y(t)=\omega(t) Y(t) \mathrm{d} t, Y(0)=y_{0}>0,
$$

define the deterministic salary process of the PPM at time $t$. In this paper, we assume that the salary process of the PPM is stochastic. In the case of deterministic salary, we shall state it.

Therefore, the flow of contributions of the PPM is given by $c Y(t)$, where $c>0$ is a proportion of PPM's salary that he/she is contributing into the scheme. We assume in this paper that $r(t), \mu(t), \sigma(t)$, $\sigma_{Y}(t), \sigma_{S}(t), \sigma_{Q}(t), \sigma_{Y 1}(t), \sigma_{Y 2}(t), \theta(t)$, $\theta_{Z}(t), \theta_{S}(t)$ are constants in time.

We now define the following exponential process which we assume to be Martingale in $P$ :

$$
Z(t)=\exp \left[-\theta^{\prime} W(t)-\frac{1}{2}\|\theta\|^{2} t\right],
$$

which will be useful in the next section.

\subsection{The Wealth Process of a PPM}

Let $X(t)$ be the wealth process and

$\Delta(t)=\left(\Delta_{Q}(t), \Delta_{S}(t)\right)$. The portfolio process, $\Delta_{Q}(t)$ represents the proportion of wealth invested in inflation-linked bond and $\Delta_{S}(t)$ the proportion of wealth invested in stock at time $t$. Then,

$$
\Delta_{0}(t)=1-\Delta_{Q}(t)-\Delta_{S}(t)
$$

is the proportion wealth invested in the riskless asset at time $t$. We now have the following definition:

Definition 1: The portfolio process $\Delta$ is said to be self-financing if the corresponding wealth process $X(t), t \in[0, T]$, satisfies

$$
\begin{aligned}
& \mathrm{d} X(t)=[(X(t)(r+\Delta(t) \lambda)+c Y(t)) \mathrm{d} t \\
& \left.+X(t)\left(\Sigma^{\prime} \Delta^{\prime}(t)\right)^{\prime} \mathrm{d} W(t)\right], X(0)=x_{0} \in \mathfrak{R}_{+},
\end{aligned}
$$

where $\lambda=\left(\sigma_{z} \theta_{z}, \mu-r\right)^{\prime}$

\section{The Expected Value of PPM's Future Contribution}

In this section, we consider the value of PPM's expected future contribution over the planning horizon. We now make the following definitions.

Definition 2: The discounted value of expected future contribution process is defined as

$$
\Phi(t)=E_{t}\left[\int_{t}^{T} \frac{\Lambda(s)}{\Lambda(t)} c Y(s) \mathrm{d} s\right],
$$

where $E_{t}(\cdot)=E(\cdot \mid F(t))$ is the conditional expectation with respect to the Brownian filtration $\{F(t)\}_{t \geq 0}$ and

$$
\Lambda(t)=Z(t) \exp (-r t)
$$

is the stochastic discount factor which adjusts for nominal interest rate and market price of risks.

Definition 3: A portfolio process is said to be admissible if the corresponding wealth process $X(t)$ satisfies

$$
\begin{aligned}
& P\left(X(t)+E_{t}\left[\int_{t}^{T} \frac{\Lambda(s)}{\Lambda(t)} c Y(s) d s\right] \geq 0\right)=1, \\
& \forall t \in[0, T] .
\end{aligned}
$$

Theorem 1: Let $\Phi(t)$ be the discounted value of expected future contribution (PVFC) process of a PPM, then

$$
\left\{\begin{array}{l}
\Phi(t)=\frac{c Y(t)}{\alpha}(\exp (\alpha(T-t))-1), \beta=0 \\
\Phi(0)=\frac{c Y_{0}}{\alpha}(\exp (\alpha T)-1), \beta=0
\end{array}\right.
$$

$$
\left\{\begin{array}{l}
\Phi(t)=-\frac{c Y(t)}{2 \sqrt{\beta}} \times \\
\left(\sqrt{\pi} \exp \left(-\frac{\alpha^{2}}{4 \beta}\right)\left(\operatorname{Erfi}\left[\frac{-2 \beta(T-t)-\alpha}{2 \sqrt{\beta}}\right]\right)+\operatorname{Erfi}\left[\frac{\alpha}{2 \sqrt{\beta}}\right]\right), \\
\beta>0 \\
\Phi(0)=-\frac{c y_{0}}{2 \sqrt{\beta}} \times \\
\left(\sqrt{\pi} \exp \left(-\frac{\alpha^{2}}{4 \beta}\right)\left(\operatorname{Erfi}\left[\frac{-2 \beta T-\alpha}{2 \sqrt{\beta}}\right]\right)+\operatorname{Erfi}\left[\frac{\alpha}{2 \sqrt{\beta}}\right]\right),
\end{array}\right.
$$

$\Phi(T)=0$, where, $\alpha=a-r-\left\|\sigma_{z}\right\|\|\theta\|$.

Proof: See Nkeki and Nwozo (2012).

Remark 1: The present value of expected future contribution of a PPM is given by $\Phi_{0}$.

In this paper, we consider the case of $\beta>0$. For the 
case of $\beta=0$, see $[4,7,10,11,12]$.

\subsection{Sensitivity Analysis of Present Value of PPM's Future Contribution (PVPPMFC)}

In this subsection, we consider the sensitivity analysis of PPM's present value future contribution for $\beta>0$ and for stochastic salary. We intend to find the elasticity of PVPPMFC with respect to change in its dependent parameters. In a sequel, we set $\Phi(0)=\Phi_{0}$. First, we find the partial derivative of $\Phi_{0}$ with respect to $\beta$.

$$
\begin{aligned}
& \frac{\partial \Phi_{0}}{\partial \beta}=\frac{c y_{0} \mathrm{e}^{\frac{-\alpha^{2}}{2 \beta}}}{4 \sqrt{\pi} \beta^{\frac{5}{2}}} \\
& \times\left[\sqrt{\beta}\left(\alpha \mathrm{e}^{\frac{3 \alpha^{2}}{4 \beta}}-\sqrt{\pi}(\alpha-2 \beta T) \mathrm{e}^{(\alpha+2 \beta T)^{2}}\right)\right. \\
& +\beta \sqrt{\pi} \mathrm{e}^{\frac{\alpha^{2}}{2 \beta}} \operatorname{Erfi}\left(\frac{\alpha}{2 \sqrt{\beta}}\right) \\
& \left.+\pi\left(\alpha^{2}-\beta\right) \operatorname{Erfi}\left(\frac{\alpha+2 \beta T}{2 \sqrt{\beta}}\right)\right] ;
\end{aligned}
$$

The partial derivative of $\Phi_{0}$ with respect to $C$ :

$$
\begin{aligned}
& \frac{\partial \Phi_{0}}{\partial c}=\frac{y_{0}}{2 \sqrt{\beta}} \\
& \times\left(\sqrt{\pi} \mathrm{e}^{\frac{-\alpha^{2}}{2 \beta}} \operatorname{Erfi}\left(\frac{\alpha+2 \beta T}{2 \sqrt{\beta}}\right)-\operatorname{Erfi}\left(\frac{\alpha}{2 \sqrt{\beta}}\right)\right) ;
\end{aligned}
$$

The partial derivative of $\Phi_{0}$ with respect to $y_{0}$ :

$$
\begin{aligned}
& \frac{\partial \Phi_{0}}{\partial y_{0}}=\frac{c}{2 \sqrt{\beta}} \\
& \times\left(\sqrt{\pi} \mathrm{e}^{\frac{-\alpha^{2}}{2 \beta}} \operatorname{Erfi}\left(\frac{\alpha+2 \beta T}{2 \sqrt{\beta}}\right)-\operatorname{Erfi}\left(\frac{\alpha}{2 \sqrt{\beta}}\right)\right)
\end{aligned}
$$

The partial derivative of $\Phi_{0}$ with respect to $a$ :

$$
\begin{aligned}
& \frac{\partial \Phi_{0}}{\partial a}=\frac{-c y_{0} \mathrm{e}^{\frac{\alpha^{2}}{2 \beta}}}{2 \sqrt{\pi} \beta^{\frac{3}{2}}} \\
& \left(\sqrt{\beta}\left(\mathrm{e}^{\frac{-3 \alpha^{2}}{4 \beta}}-\sqrt{\pi} \mathrm{e}^{\frac{(\alpha+2 \beta T)^{2}}{4 \beta}}\right)-\alpha \pi E r f i\left(\frac{-\alpha-2 \beta T}{2 \sqrt{\beta}}\right)\right) ;
\end{aligned}
$$

The partial derivative of $\Phi_{0}$ with respect to $T$ :

$$
\frac{\partial \Phi_{0}}{\partial T}=c y_{0} \mathrm{e}^{\alpha T+-\frac{\alpha^{2}}{4 \beta}+\beta T^{2}}
$$

The partial derivative of $\Phi_{0}$ with respect to $r, \theta_{z}, \theta_{S}, \sigma_{Y 1}, \sigma_{Y 2}$ are respectively given as;

$$
\begin{aligned}
& \frac{\partial \Phi_{0}}{\partial r}=\frac{-c y_{0}}{2 \beta^{\frac{3}{2}}}\left(1-\frac{\sigma_{Y 2}}{\sigma_{S} \sqrt{1-\rho^{2}}}\right) \\
& \times \mathrm{e}^{\frac{-\alpha^{2}}{2 \beta}}\left(\sqrt{\beta} \mathrm{e}^{\frac{(\alpha+2 \beta T)^{2}}{4 \beta}}-\sqrt{\frac{\beta}{\pi}} \mathrm{e}^{\frac{3 \alpha^{2}}{4 \beta}}+\alpha \sqrt{\pi} \operatorname{Erfi}\left(\frac{-\alpha-2 \beta T}{2 \sqrt{\beta}}\right)\right)
\end{aligned}
$$

$$
\begin{aligned}
& \frac{\partial \Phi_{0}}{\partial \theta_{z}}=\frac{-c y_{0}}{2 \beta^{\frac{3}{2}}}\left(\sigma_{Y 1}-\frac{\rho \sigma_{Y 2}}{\sqrt{1-\rho^{2}}}\right) \\
& \times \mathrm{e}^{\frac{-\alpha^{2}}{2 \beta}}\left(\sqrt{\beta} \mathrm{e}^{\frac{(\alpha+2 \beta T)^{2}}{4 \beta}}-\sqrt{\frac{\beta}{\pi}} \mathrm{e}^{\frac{3 \alpha^{2}}{4 \beta}}+\alpha \sqrt{\pi} \operatorname{Erfi}\left(\frac{-\alpha-2 \beta T}{2 \sqrt{\beta}}\right)\right)
\end{aligned}
$$

$$
\begin{aligned}
& \frac{\partial \Phi_{0}}{\partial \sigma_{Y 1}}=\frac{c y_{0} \theta_{2} \mathrm{e}^{\frac{\alpha^{2}}{2 \beta}}}{2 \beta^{\frac{3}{2}} \sigma_{S} \sqrt{\pi\left(1-\rho^{2}\right)}} \\
& \times\left(\sigma_{S} \sqrt{\beta\left(1-\rho^{2}\right)}\left(\mathrm{e}^{\frac{3 \alpha^{2}}{4 \beta}}-\sqrt{\pi} \mathrm{e}^{\frac{3(\alpha+2 \beta T)^{2}}{4 \beta}}\right)+\alpha \pi E r f i\left(\frac{\alpha+2 \beta T}{2 \sqrt{\beta}}\right)\right)
\end{aligned}
$$

$$
\begin{aligned}
& \frac{\partial \Phi_{0}}{\partial \sigma_{Y 2}}=-\frac{c y_{0} \theta_{S}}{2 \beta^{\frac{3}{2}}} \mathrm{e}^{\frac{-\alpha^{2}}{2 \beta}} \\
& \times\left(\sqrt{\beta} \mathrm{e}^{\frac{(\alpha+2 \beta T)^{2}}{4 \beta}}-\sqrt{\frac{\beta}{\pi}} \mathrm{e}^{\frac{3 \alpha^{2}}{4 \beta}}+\alpha \sqrt{\pi} \operatorname{Erfi}\left(\frac{-\alpha-2 \beta T}{2 \sqrt{\beta}}\right)\right) . \\
& \frac{\partial \Phi_{0}}{\partial \mu}=\frac{-c y_{0} \sigma_{Y 2} \mathrm{e}^{\frac{-\alpha^{2}}{2 \beta}}}{2\left(\rho^{2}-1\right) \sigma_{S}^{2} \sqrt{\pi} \beta^{\frac{3}{2}}} \\
& \times\left(\sigma_{S} \sqrt{\beta} \sqrt{1-\rho^{2}}\left(\mathrm{e}^{\frac{3 \alpha^{2}}{4 \beta}}-\sqrt{\pi} \mathrm{e}^{\frac{(\alpha+2 \beta T)^{2}}{4 \beta}}\right)+\pi \alpha \operatorname{Erfi}\left(\frac{\alpha+2 \beta T}{2 \sqrt{\beta}}\right)\right) . \\
& \frac{\partial \Phi_{0}}{\partial \rho}=\frac{-c y_{0}}{2 \sqrt{\beta}}\left(\frac{\sigma_{Y 2} \theta_{z}}{\sqrt{1-\rho^{2}}}-\frac{\rho \theta_{S} \sigma_{Y 2}}{1-\rho^{2}}\right) \\
& \times\left(\frac{\mathrm{e}^{\frac{\alpha^{2}}{4 \beta}}}{\sqrt{\beta \pi}}-\frac{\mathrm{e}^{\frac{-\alpha^{2}}{2 \beta}-\frac{(\alpha-2 \beta T)^{2}}{4 \beta}}}{\sqrt{\beta}}-\frac{\alpha \sqrt{\pi} \mathrm{e}^{\frac{-\alpha^{2}}{2 \beta}}}{\beta} \operatorname{Erfi}\left(\frac{-\alpha-2 \beta T}{2 \sqrt{\beta}}\right) .\right.
\end{aligned}
$$

We set (to obtain the values in Table 1 by varying each of the parameters) $a=0.0292, r=0.04$, $\sigma_{Y 1}=0.18, \quad \sigma_{Y 2}=0.20, \theta_{z}=0.09, \quad \sigma_{S}=0.30$, $\rho=0.40, \quad \mu=0.09, \quad \beta=0.01, \quad T=20, \sigma_{Q}=0.25$, 
$y_{0}=0.8, c=0.15$.

Table 1 gives the values of the parameters and the corresponding change in PVPPMFC. It is observed that the PVPPMFC is positively sensitive to all the parameters except $c$ and $y_{0}$ that is constant overtime (or we say that PVPPMFC is inelastic in $c$ and $y_{0}$ ). But, PVPPMFC is negatively sensitive in $\rho$. We observe that as $\rho$ increases by 0.1 , the PVPPMFC decreases by about 15.192. We also observed that among the sensitive parameters, some are more sensitive than the others. For instance, a small change in $\beta$ will lead to a very high proportionate change in PVPPMFC, i.e., an increase of $\beta$ by 0.001 unit will bring about an average increase of PVPPMFC by 251 units. It is further observed that an increase of $T$ by one unit will lead to a very small change in PVPPMFC. Increasing $a$ by 0.001 will lead to about an average of 2.19 increases in PCPPMFC. Similarly, an increase in $r$ by 0.01 will lead to a proportionate increase in PVPPMFC by 1.65 . Similarly, an increase in $\theta_{z}$ by 0.01 will bring about 0.20 proportionate increases in PVPPMFC. Again, an increase in $\sigma_{Y 1}$ by 0.05 will lead to about 0.75 proportionate increases in PVPPMFC. Finally, an increase in $\sigma_{Y 2}$ by 0.05 will lead to about 1.52 proportionate increases in PVPPMFC. When increase by 0.01 , the PVPPMFC increases alongside with 15.2. We therefore conclude that PVPPMFC is elastic with respect to change in the parameters. The elasticity of PVPPMFC with respect to change in the above parameters will enable fund managers and investors to value assets, flow of contributions, and investment flow that are to be receive some time in future. From (14), we obtain the critical time horizon for the present value of PPM's future discounted contribution as follows:

$$
T^{*}=-\frac{1}{2}\left(\frac{\alpha}{\beta} \mp \sqrt{\frac{\alpha^{2}}{\beta^{2}}-4 \log _{\mathrm{e}} c y_{0}-\frac{\alpha^{2}}{\beta}}\right) .
$$

Lemma 1: The dynamics of the discounted value of future contribution process is given by

$$
\begin{aligned}
& \mathrm{d} \Phi(t)=\Phi(t)\left(\kappa(t) \mathrm{d} t+\sigma_{z} \mathrm{~d} W(t)\right) \\
& { }_{-c} Y(t) \mathrm{d} t
\end{aligned}
$$

where,

$$
\begin{gathered}
A(t)=\sqrt{\pi} \\
\left(\operatorname{Erfi}\left[\frac{-2 \beta(T-t)-\alpha}{2 \sqrt{\beta}}\right]+\operatorname{Erfi}\left[\frac{\alpha}{2 \sqrt{\beta}}\right]\right), \\
\kappa(t)=-\frac{((a+\beta t) A(t)+H(t))}{A(t)}, T>t, \\
H(t)=\exp \left(\frac{(-2 \beta(T-t)-\alpha)^{2}}{4 \beta}\right)-2 \sqrt{\beta} \exp \left(\frac{\alpha^{2}}{4 \beta}\right) .
\end{gathered}
$$

Proof: See Nkeki and Nwozo (2012).

\subsection{Value of PPM's Wealth Process}

Let the value of PPM's wealth be $V(t)$ at time $t$ is given by

$$
V(t)=X(t)+\Phi(t), t \in[0, T] .
$$

Finding the differential of both sides of (21) and sub-

\begin{tabular}{|c|c|c|c|c|c|c|c|c|c|c|c|c|c|c|c|c|c|}
\hline$T$ & $\frac{\partial \Phi_{0}}{\partial T}$ & $\beta$ & $\frac{\partial \Phi_{0}}{\partial \beta}$ & $a$ & $\frac{\partial \Phi_{0}}{\partial a}$ & $r$ & $\frac{\partial \Phi_{0}}{\partial r}$ & $\theta_{z}$ & $\frac{\partial \Phi_{0}}{\partial \theta_{z}}$ & $\sigma_{Y 1}$ & $\frac{\partial \Phi_{0}}{\partial \sigma_{Y 1}}$ & $\sigma_{Y 2}$ & $\frac{\partial \Phi_{0}}{\partial \sigma_{Y 2}}$ & $\mu$ & $\frac{\partial \Phi_{0}}{\partial \mu}$ & $\rho$ & $\frac{\partial \Phi_{0}}{\partial \rho}$ \\
\hline 1 & 0.1062 & 0.001 & -2838.87 & 0.020 & 116.76 & 0.01 & -42.83 & 0.05 & -13.519 & 0.15 & -12.903 & 0.20 & -19.52 & 0.01 & -244.9 & 0.1 & 1.93 \\
\hline 2 & 0.1035 & 0.002 & -60.0597 & 0.021 & 118.84 & 0.02 & -40.93 & 0.06 & -13.310 & 0.20 & -11.952 & 0.25 & -17.26 & 0.02 & -220.9 & 0.2 & 1.60 \\
\hline 3 & 0.1030 & 0.003 & 249.936 & 0.022 & 120.94 & 0.03 & -39.10 & 0.07 & -13.103 & 0.25 & -11.057 & 0.30 & -15.21 & 0.03 & -198.8 & 0.3 & 1.25 \\
\hline 5 & 0.1081 & 0.005 & 483.543 & 0.024 & 125.23 & 0.05 & -35.63 & 0.09 & -12.697 & 0.35 & -09.425 & 0.40 & -11.69 & 0.05 & -159.7 & 0.5 & 0.28 \\
\hline 6 & 0.1141 & 0.006 & 627.521 & 0.025 & 127.42 & 0.06 & -33.99 & 0.10 & -12.497 & 0.40 & -08.684 & 0.45 & -10.18 & 0.06 & -142.5 & 0.6 & -0.54 \\
\hline 7 & 0.1230 & 0.007 & 824.141 & 0.026 & 129.64 & 0.07 & -32.41 & 0.11 & -12.300 & 0.45 & -07.989 & 0.50 & -08.82 & 0.07 & -126.8 & 0.7 & -1.99 \\
\hline 8 & 0.1351 & 0.008 & 1096.40 & 0.027 & 131.89 & 0.08 & -30.89 & 0.12 & -12.106 & 0.50 & -07.337 & 0.55 & -07.60 & 0.08 & -112.6 & 0.8 & -5.22 \\
\hline 9 & 0.1515 & 0.009 & 1474.95 & 0.028 & 134.17 & 0.09 & -29.42 & 0.13 & -11.914 & 0.55 & -06.727 & 0.60 & -06.52 & 0.09 & -99.6 & 0.9 & -16.45 \\
\hline 10 & 0.1733 & 0.010 & 2002.66 & 0.029 & 136.48 & 0.10 & -28.01 & 0.14 & -11.724 & 0.60 & -06.157 & 0.65 & -05.55 & 0.10 & -87.9 & 0.99 & -134.8 \\
\hline
\end{tabular}
stituting in (6) and (20), we obtain

$$
\begin{aligned}
& \mathrm{d} V(t)=[X(t)(r+\Delta(t) \lambda)+\Phi(t) \kappa(t)] \mathrm{d} t \\
& +\left(\sigma_{Y}^{\prime} \Phi(t)+\left(\Sigma^{\prime} \Delta^{\prime}(t)\right) X(t)\right)^{\prime} \mathrm{d} W(t) .
\end{aligned}
$$

\section{The Optimization Process and Portfolio Value of a PPM}

In this section, we consider the optimal portfolio process.

Table 1. Simulation of the sensitivity analysis of PVPPMFC. 
We define the general objective function

$$
J(t, V, \Delta)=E[u(V(T)) \mid X(t)=x, \Phi(t)=\Phi]
$$

where $u(V(t))$ is the path of $V(t)$ given the portfolio strategy $\Delta$. Define $\Pi(V)$ to be the set of all admissible portfolio strategy that are $F_{V}$ - progressively measurable, and let $u(V(t))$ be a concave value function in $V(t)$ such that

$$
\begin{aligned}
& U(V(t)) \\
& =\sup _{\Delta \in \Pi(V)} E[u(V(T)) \mid X(t)=x, \Phi(t)=\Phi] \\
& =\sup _{\Delta \in \Pi(V)} J(t, V, \Delta) .
\end{aligned}
$$

Then $U(V(t))$ satisfies the HJB equation

$$
U_{t}+\sup _{\Delta \in \Pi(V)} H(t, v, \Delta)=0
$$

subject to:

$$
U(T, v)=\frac{v^{\gamma}}{\gamma}, \gamma>0
$$

where,

$$
\begin{aligned}
& H(t, v, \Delta)=(r x+x \Delta(t) \lambda) U_{x}+\Phi \kappa(\tau) U_{\Phi} \\
& +\frac{1}{2} x^{2}\left(\Sigma^{\prime} \Delta^{\prime}(t)\right)^{\prime} \Sigma^{\prime} \Delta^{\prime}(t) U_{x x} \\
& +x \Phi \sigma_{Y} \Sigma \Delta(t) U_{x \Phi}+\frac{1}{2} \Phi^{2} \sigma_{Y} \sigma_{Y}^{\prime} U_{\Phi \Phi} .
\end{aligned}
$$

Finding the partial derivative of $H(t, v, \Delta)$ with respect to the optimal control $\Delta(t)$ and setting it to zero, we obtain

$$
\Delta^{* \prime}(t)=\frac{-\left(\Sigma \Sigma^{\prime}\right)^{-1}\left(\lambda U_{x}+\Phi \Sigma \sigma_{Y}^{\prime} U_{x \Phi}\right)}{x U_{x x}} .
$$

Substituting (24) into (23), we obtain the HJB equation (25), we have

$$
\begin{aligned}
& U_{t}+r x U_{x}+\Phi \kappa U_{\Phi}-\frac{1}{2} \frac{(M \lambda)^{\prime} \lambda U_{x}^{2}}{U_{x x}} \\
& +\frac{M^{\prime}\left(\Sigma \sigma_{Y}^{\prime}\right)^{\prime} \lambda \Phi U_{x} U_{x \Phi}}{U_{x x}}-\frac{\Phi^{2} \sigma_{Y} \sigma_{Y}^{\prime} U_{x \Phi}^{2}}{2 U_{x x}} \\
& +\frac{1}{2} \Phi^{2} \sigma_{Y} \sigma_{Y}^{\prime} U_{\Phi \Phi}=0 .
\end{aligned}
$$

where $M=\left(\Sigma \Sigma^{\prime}\right)^{-1}$.

Proposition: Let

$$
U(t, v)=\frac{(v R(t))^{\gamma}}{\gamma}, \gamma>0,
$$

be the solution to the HJB Equation (25), then,

$$
\begin{aligned}
& R^{\prime}(t)+r x v^{-1} R(t)+\frac{1}{2} \frac{(M \lambda)^{\prime} \lambda}{\gamma-1} R(t) \\
& +\Phi \kappa(\tau) v^{-1} R(t)-\Phi M^{\prime}\left(\Sigma \sigma_{Y}^{\prime}\right)^{\prime} \lambda v^{-1} R(t)=0 ; \\
& \Delta^{* \prime}(t)=\frac{M \lambda\left(X^{*}(t)+\Phi(t)\right)}{(1-\gamma) X^{*}(t)}-\frac{\Phi(t) M \Sigma \sigma_{Y}^{\prime}}{X^{*}(t)} .
\end{aligned}
$$

Proof: We commence by obtaining the following partial derivatives:

$$
\begin{gathered}
U_{t}=v^{\gamma} R(t)^{\gamma-1} R^{\prime}(t) \\
U_{x}=v^{\gamma-1} R(t)^{\gamma} \\
U_{x x}=(\gamma-1) v^{\gamma-2} R(t)^{\gamma} \\
U_{x \Phi}=(\gamma-1) v^{\gamma-2} R(t)^{\gamma} \\
U_{\Phi}=v^{\gamma-1} R(t)^{\gamma} \\
U_{\Phi \Phi}=(\gamma-1) v^{\gamma-2} R(t)^{\gamma}
\end{gathered}
$$

Substituting the partial derivatives above into (25), we obtain

$$
\begin{aligned}
& R^{\prime}(t)+r x v^{-1} R(t)+\frac{1}{2} \frac{(M \lambda)^{\prime} \lambda}{\gamma-1} R(t) \\
& +\Phi \kappa(\tau) v^{-1} R(t)-\Phi M^{\prime}\left(\Sigma \sigma_{Y}^{\prime}\right)^{\prime} \lambda v^{-1} R(t)=0 .
\end{aligned}
$$

Again, substituting the partial derivatives into (24), we obtain

$$
\Delta^{* \prime}(t)=\frac{M \lambda\left(X^{*}(t)+\Phi(t)\right)}{(1-\gamma) X^{*}(t)}-\frac{\Phi(t) M \Sigma \sigma_{Y}^{\prime}}{X^{*}(t)} .
$$

Therefore, the portfolio value in the riskless asset is obtain as

$$
\Delta_{0}^{* \prime}(t)=1+\frac{\Phi(t) M \Sigma \sigma_{Y}^{\prime}}{X^{*}(t)}-\frac{M \lambda\left(X^{*}(t)+\Phi(t)\right)}{(1-\gamma) X^{*}(t)} .
$$

The portfolio value (27) is made up of two parts. The first part is the OVMP value and the second part is the intertemporal hedging term that offset any shocks to the stochastic salary of the PPM at time $t$. It is observe in (28) that the flow of intertemporal term is a gradual transfer fund from the risky assets to the riskless one over time. The intertemporal hedging term, interestingly is a function of financial market behaviour and volatility vector of the effective salary process of a PPM. It is also a ratio of the PPM discounted value of expected future contributions of a PPM to the optimal wealth process. This strategy is strongly recommended for PPMs of whom contributions are made compulsory. This will ensure that the inflation risks in the portfolio of members are hedged. We therefore say that (27) is a portfolio with 
inflation protection strategy. The solution to (26) can be obtained numerically.

\section{Numerical Example}

Let $a=0.0292, r=0.04, \sigma_{Y 1}=0.18, \sigma_{Y 2}=0.20$, $\theta_{z}=0.09, \sigma_{S}=0.30, \rho=0.40, \mu=0.09, \beta=0.01$, $\gamma=0.80, T=20, \sigma_{Q}=0.25, y_{0}=0.8, c=0.15$, we have the following figures:

Figures 1-3 are obtained for $\beta=0$ and for deterministic salary process. Figure 1 shows the portfolio value of a PPM invested in inflation-linked bond for a period of 20 years given that the wealth is between 1 to 6 million. Similarly, Figure 2 shows the portfolio value of a PPM invested in stock for the same period and the same wealth. Figure 3 shows the portfolio value of a PPM in cash account. It is observed that the portfolio values in stock and inflation-linked bond are nonnegative, while in cash account is negative over time. We therefore recommend (based on these numerical examples) that the portfolio value in cash account should be shorten by the negative value to finance the risky assets (i.e., more money should be borrowed from cash account to finance the investment in stock and inflation-linked bond). It is also observed that the wealth generated by stock is higher than that of inflation-linked bond. This suggest that more fund should be invested in stock that inflation-linked bond to ensure maximum returns for members at retirement. Figure 4 shows the portfolio value in stock when $\beta=0$, and for the case salary stochastic. Figure 5 shows the portfolio value in Stock when $\beta=0$, for salary stochastic and randomness is relaxed. Figure 6 shows the portfolio value in cash account, when $\beta=0$, and salary stochastic. Figure 7 shows the portfolio value in cash account, when $\beta=0$, salary stochastic and randomness is relaxed. Figure 8 shows the portfolio value in inflation-linked bond, when $\beta=0$, and salary is stochastic. Figure 9 shows portfolio value in inflation-linked bond for $\beta=0$, salary stochastic and randomness is relaxed.

Figures 4, 6, 8 are established for $\beta=0$ and for stochastic salary process. These figures are made up of several shocks and paths. These shock paths made it difficult to make useful decisions, hence the need for us to relax the randomness associated with the portfolios for effective decision making. We now have that the following Figures 5, 7, 9 are special cases of Figures 4, 6, 8, respectively. Similar behaviors exhibited by Figures 1-3 were also exhibited by Figures 5, 7, 9. The major difference is that the portfolio value under stochastic salary yields higher returns that the deterministic salary. This makes sense, since it is expected that, the higher the risk taken, the higher the expected wealth. Interestingly, from the numerical examples, the amount that was gradually transferred from the risky assets to cash account seems to have been re-invested back to the risky assets overtime.
Figures 10-12 show the portfolio values for deterministic salary and for $\beta>0$ in inflation-linked bond, stock and cash account, respectively. We observed that under this strategy, the entire fund should be invested in stock for maximum wealth for the PPM at retirement.

Figures 13-15 show the portfolio values for stochastic salary and $\beta>0$ in inflation-linked bond, stock and cash account overtime. It should be noted that the shocks associated with the portfolios arise from the salary risks of the PPM overtime.

\section{Expected Wealth and Variance of the Expected Wealth}

In other to determine the expected wealth of the PPM at time $t$, we find the mathematical expectation of (22) as follows:

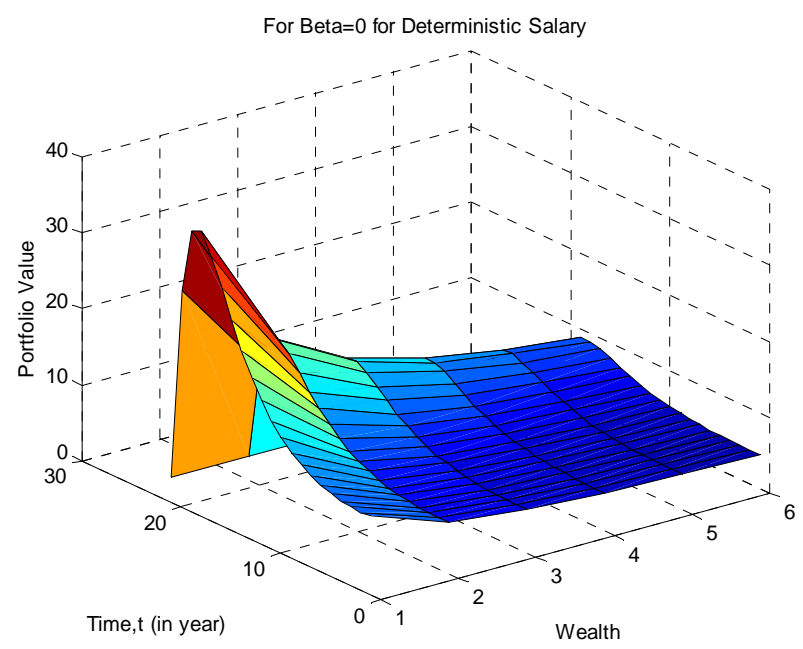

Figure 1. The portfolio value in inflation-linked bond for $\beta=0$ and salary deterministic.

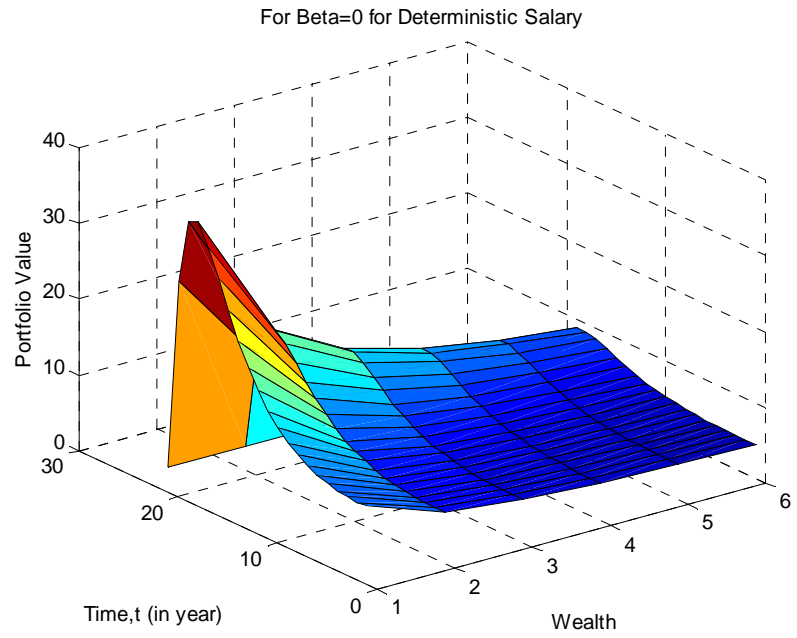

Figure 2. The portfolio value of a PPM in stock for $\beta=0$ and salary deterministic. 


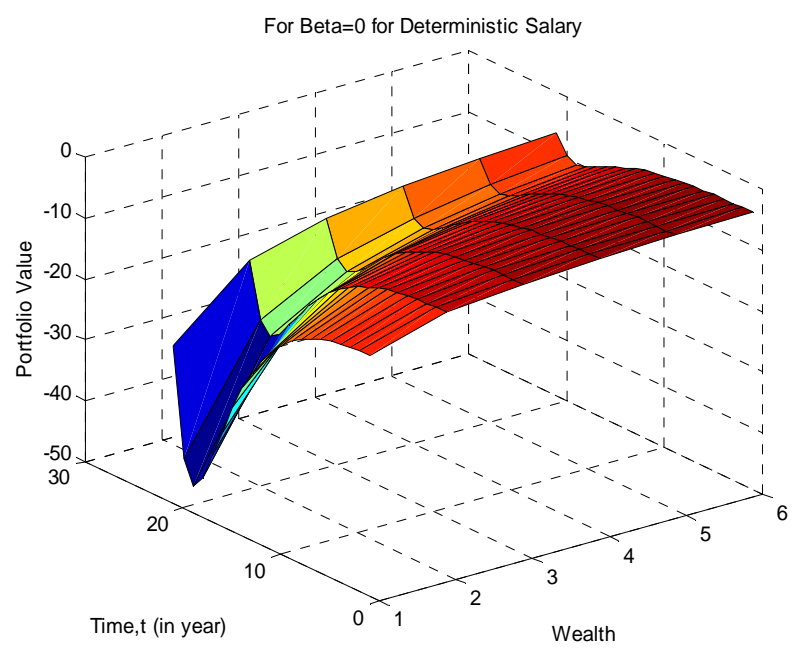

Figure 3. The portfolio value of a PPM in cash account for $\beta=0$ and salary deterministic.

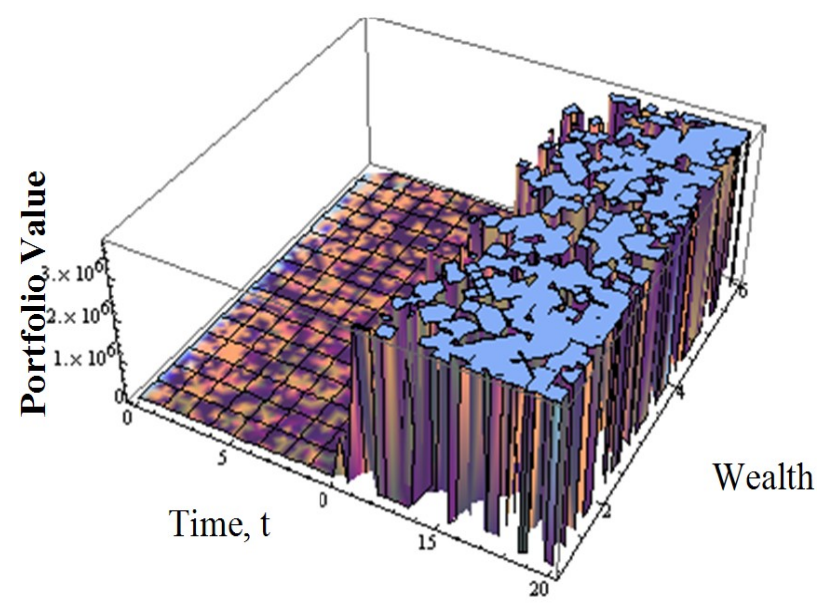

Figure 4. The portfolio value in stock. When $\beta=0$, and salary stochastic.

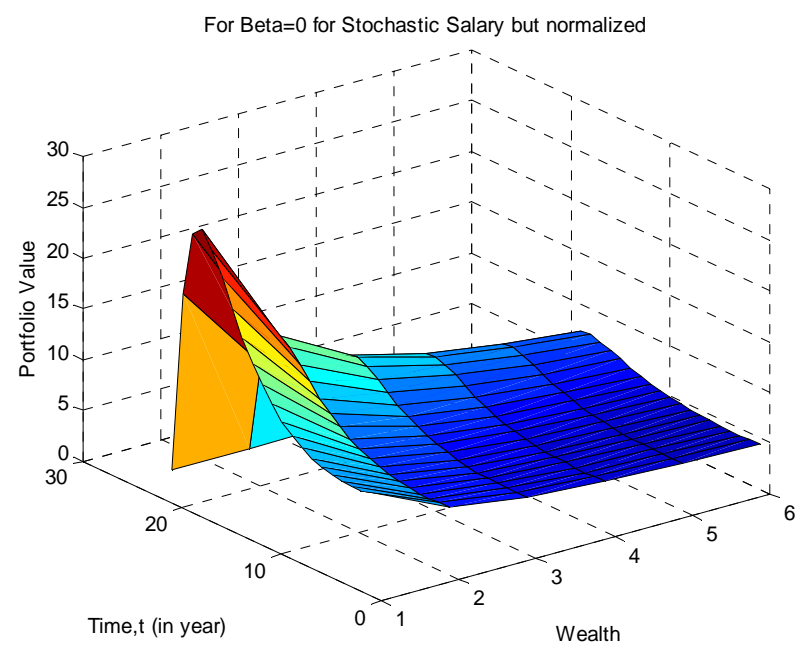

Figure 5. The portfolio value in stock. When $\beta=0$, and salary stochastic and randomness is relaxed.

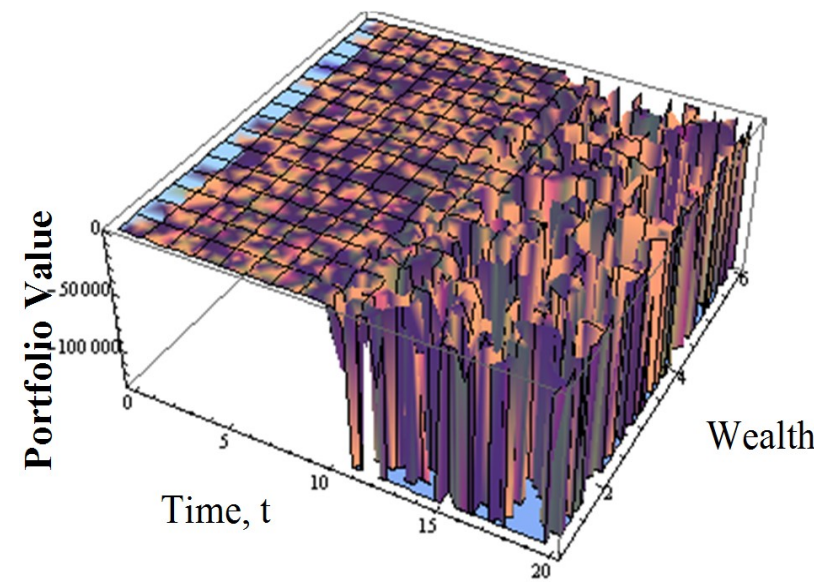

Figure 6. The portfolio value in cash account. When $\beta=0$, and salary stochastic.

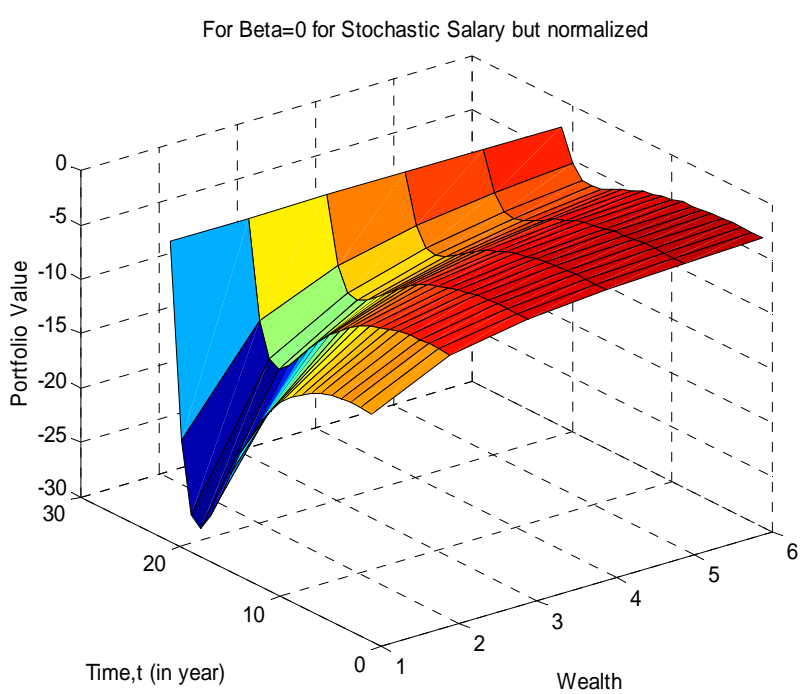

Figure 7. The portfolio value in cash account. When $\beta=0$, and salary stochastic randomness is relaxed.

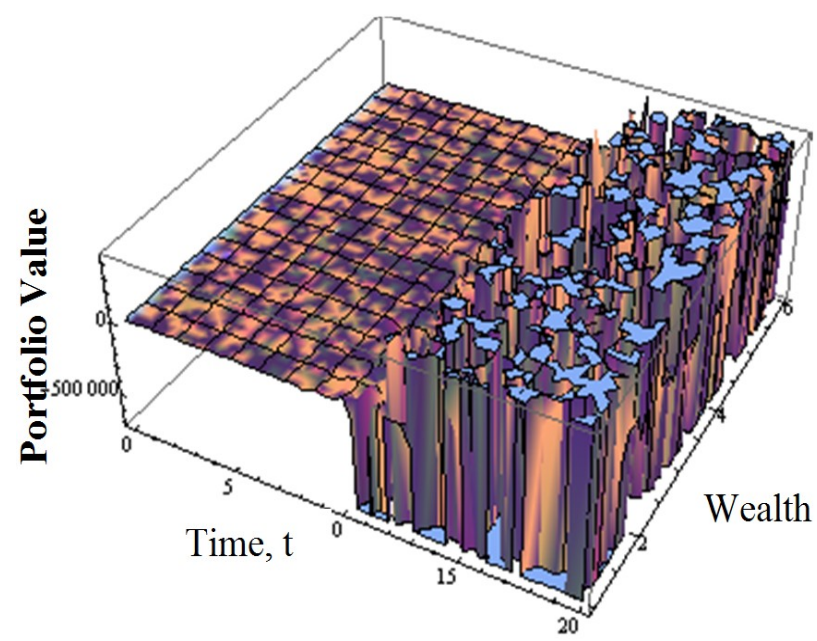

Figure 8. The portfolio value in inflation-linked bond. when $\beta=0$, and salary stochastic. 


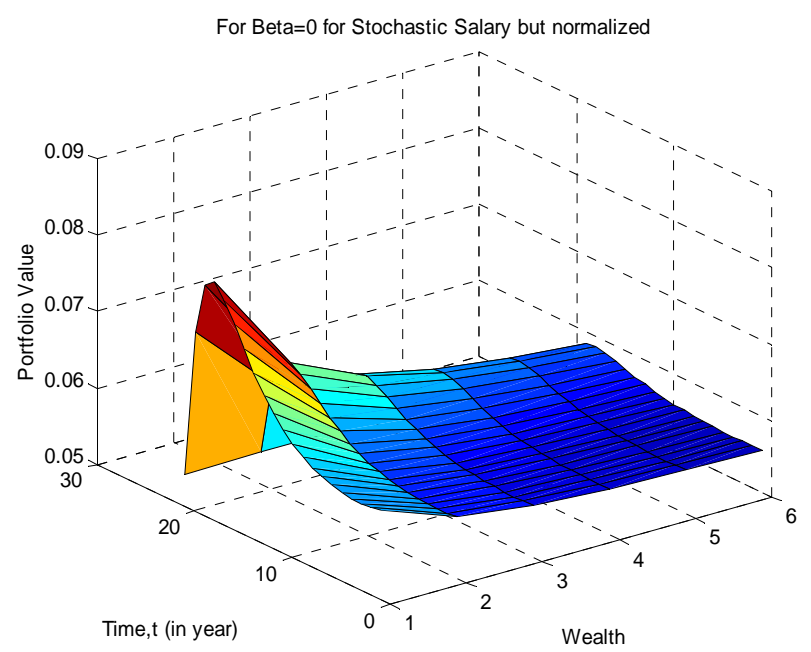

Figure 9. Portfolio value in inflation-linked bond. When $\beta=0$, and salary stochastic and randomness is relaxed.

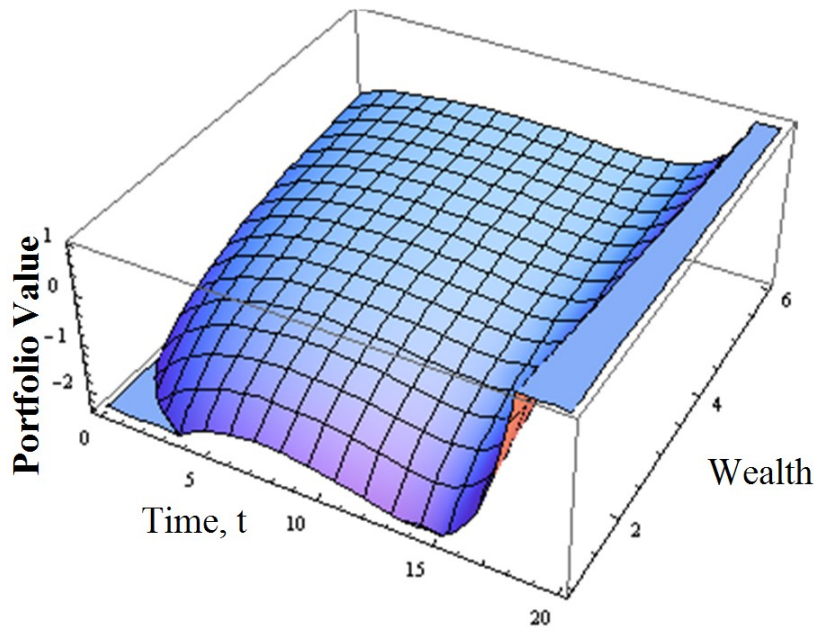

Figure 10. The portfolio value in inflation-linked bond for $\beta>0$ and deterministic salary.

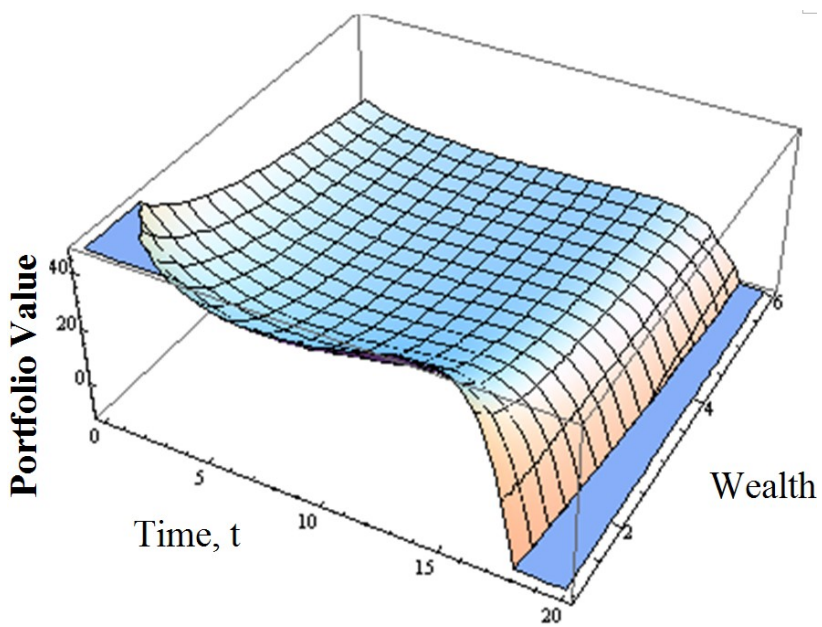

Figure 11. The portfolio value in stock for $\beta>0$ and deterministic salary.

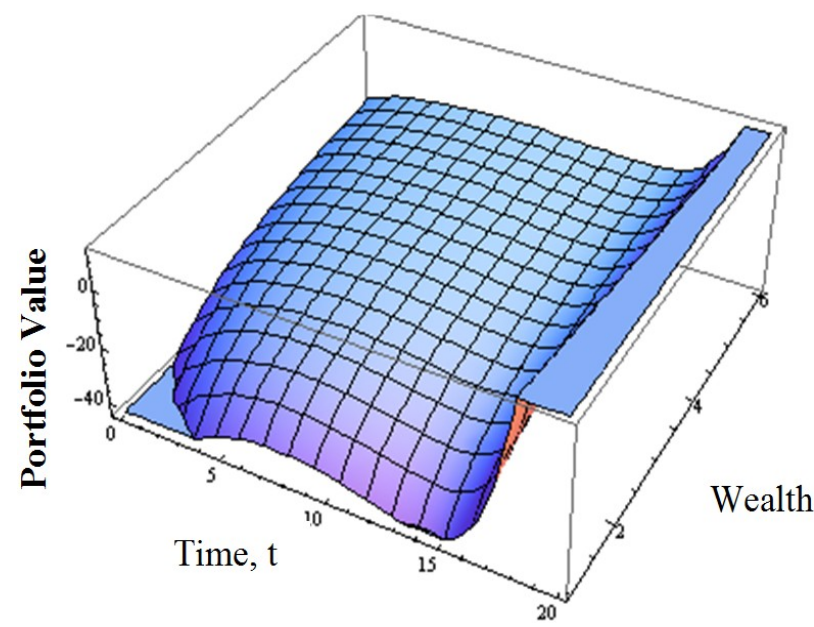

Figure 12. The portfolio value in cash account for $\beta>0$ and deterministic salary.

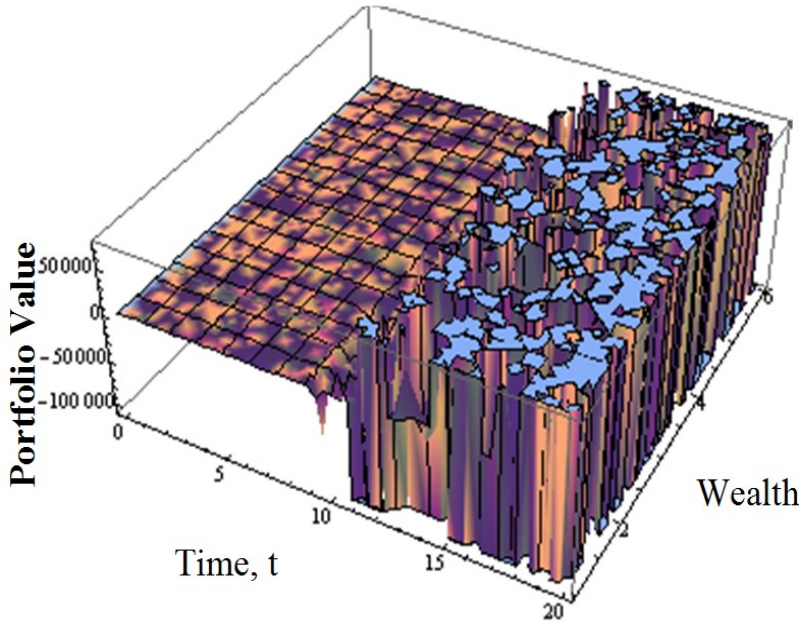

Figure 13. The portfolio value in inflation-linked bond for $\beta>0$ and for stochastic salary.

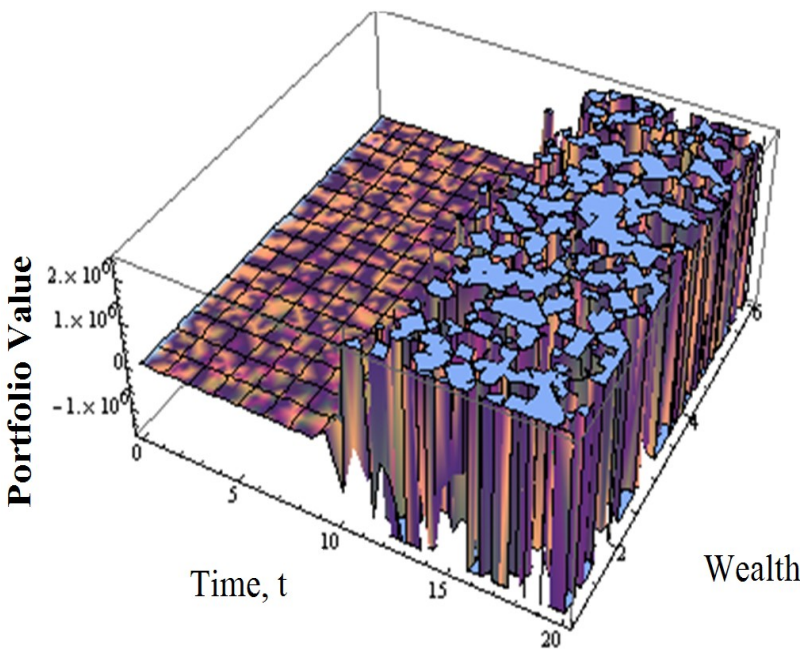

Figure 14. The portfolio value in stock for $\beta>0$ and salary stochastic. 


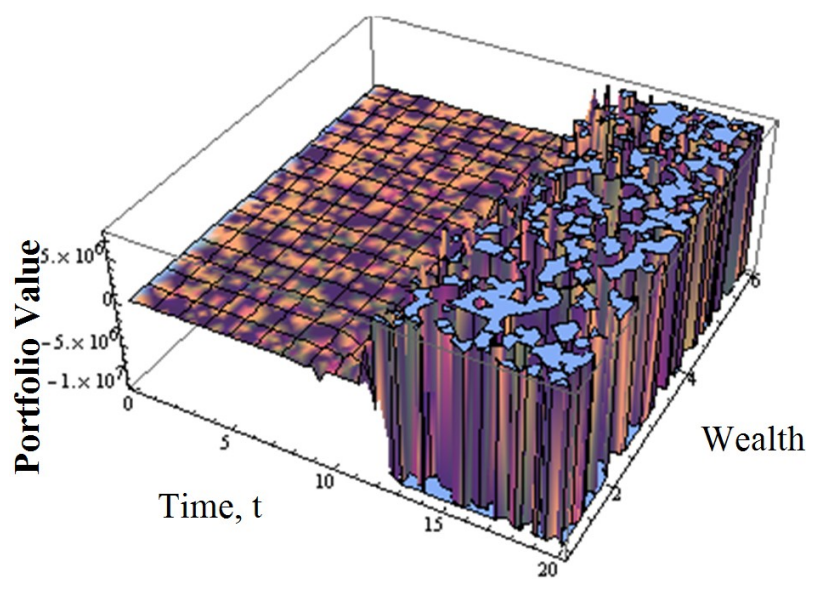

Figure 15. The portfolio value in cash account for $\beta>0$ and salary stochastic.

$$
\begin{aligned}
& \mathrm{d} V^{*}(t) \\
= & \left(r V^{*}(t)+\frac{(M \lambda)^{\prime} \lambda V^{*}(t)}{1-\gamma}\right. \\
& \left.-\left(r+\left(M \Sigma^{\prime} \sigma_{Y}^{\prime}\right)^{\prime} \lambda-\kappa(t)\right) \Phi(t)\right) \mathrm{d} t \\
& +\frac{(\Sigma M \lambda)^{\prime} V^{*}(t)}{1-\gamma} \mathrm{d} W(t) .
\end{aligned}
$$

Taking the mathematical expectation (29), we have

$$
\begin{aligned}
& \mathrm{d} E\left(V^{*}(t)\right) \\
& =\left(\left(r+\frac{(M \lambda) \lambda}{1-\gamma}\right) E\left(V^{*}(t)\right)\right. \\
& \left.-\left(r+\left(M \Sigma^{\prime} \sigma_{Y}^{\prime}\right)^{\prime} \lambda-\kappa(t)\right) E(\Phi(t))\right) \mathrm{d} t
\end{aligned}
$$

Solving (30), we have

$$
E\left(V^{*}(t)\right)=v_{0} \mathrm{e}^{\delta t}-\int_{0}^{t} \mathrm{e}^{\delta(t-s)} f(s) \mathrm{d} s
$$

where,

$$
\begin{aligned}
& f(s)=\left(r+\left(M \Sigma \sigma_{Y}^{\prime}\right)^{\prime} \lambda-\kappa(s)\right) E(\Phi(s)), \\
& \delta=r+\frac{(M \lambda)^{\prime} \lambda}{1-\gamma} \\
& \mathrm{d} V^{*_{2}}(t) \\
& =\left(\left(2 \delta+\frac{(\Sigma M \lambda)^{\prime} \Sigma M \lambda}{(1-\gamma)^{2}}\right) V^{* 2}(t)-2 f(t) V^{*}(t)\right) \mathrm{d} t \\
& +\frac{2(\Sigma M \lambda)^{\prime} V^{* 2}(t)}{1-\gamma} \mathrm{d} W(t)
\end{aligned}
$$

Taking the mathematical expectation of (32), we have

$$
\begin{aligned}
& \mathrm{d} E\left(V^{* 2}(t)\right) \\
& =\left(\left(2 \delta+\frac{(\Sigma M \lambda)^{\prime} \Sigma M \lambda}{(1-\gamma)^{2}}\right) \times E\left(V^{* 2}(t)\right)-2 f(t) E\left(V^{*}(t)\right)\right) \mathrm{d} t
\end{aligned}
$$

Integrating (33), we have

$$
\begin{aligned}
& E\left(V^{* 2}(t)\right)=v_{0}^{2} \mathrm{e}^{\eta t}-v_{0} \int_{0}^{t} \mathrm{e}^{\eta t} \mathrm{e}^{(\delta-\eta) s} f(s) \mathrm{d} s \\
& +\int_{0}^{t} \int_{0}^{s} \mathrm{e}^{\eta(t-s)} f(s) f(\tau) \mathrm{e}^{\delta(T-\tau)} \mathrm{d} \tau \mathrm{d} s,
\end{aligned}
$$

where,

$$
\eta=2 \delta+\frac{(\Sigma M \lambda)^{\prime} \Sigma M \lambda}{(1-\gamma)^{2}} .
$$

\section{Efficient Frontier}

In this subsection, we presents the efficient frontier of the three classes of assets.

At $t=T$, we have

$$
\begin{aligned}
& E\left(V^{*}(T)\right)=v_{0} \mathrm{e}^{\delta T}+\phi_{1}(T) \\
& E\left(V^{* 2}(T)\right)=v_{0}^{2} \mathrm{e}^{\eta T}+\phi_{2}(T)
\end{aligned}
$$

where

$$
\begin{gathered}
\phi_{1}(T)=-\int_{0}^{T} \mathrm{e}^{\delta(T-s)} f(s) \mathrm{d} s, \\
\phi_{2}(T)=-v_{0} \int_{0}^{T} \mathrm{e}^{\eta T} \mathrm{e}^{(\delta-\eta) s} f(s) \mathrm{d} s \\
+\int_{0}^{T} \int_{0}^{s} \mathrm{e}^{\eta(T-s)} f(s) f(\tau) \mathrm{e}^{\delta(T-\tau)} \mathrm{d} \tau \mathrm{d} s
\end{gathered}
$$

Therefore, the variance of the portfolio is obtained as

$$
\begin{aligned}
\operatorname{Var}\left(V^{*}(T)\right) & =E\left(V^{* 2}(T)\right)-\left(E\left(V^{*}(T)\right)\right)^{2} \\
& =v_{0}^{2} \mathrm{e}^{\eta T}+\phi_{2}(T)-\left(E\left(V^{*}(T)\right)\right)^{2} .
\end{aligned}
$$

(34) is the expected terminal wealth of the PPM and (35) is variance of the expected terminal wealth of the PPM. The efficient frontier of the three classes of assets is obtain as

$$
\begin{aligned}
\operatorname{Var}\left(V^{*}(T)\right)= & v_{0}^{2} \mathrm{e}^{\eta T}+\phi_{2}(T)-\left(E\left(V^{*}(T)\right)\right)^{2} \\
& =\left(v_{0} \mathrm{e}^{\frac{1}{2} \eta T}-E\left(V^{*}(T)\right)\right)^{2}+\phi_{2}(T) \\
& -2\left(v_{0} \mathrm{e}^{\frac{1}{2} \eta T}-E\left(V^{*}(T)\right)\right)^{2}+2 v_{0}^{2} \mathrm{e}^{\eta T} \\
& =-\left(v_{0} \mathrm{e}^{\frac{1}{2} \eta T}-E\left(V^{*}(T)\right)\right)^{2}+\phi_{2}(T)+2 v_{0}^{2} \mathrm{e}^{\eta T} \\
& =F(T)-\left(E\left(V^{*}(T)\right)-v_{0} \mathrm{e}^{\frac{1}{2} \eta T}\right)^{2},
\end{aligned}
$$


where,

$$
F(T)=2 v_{0}^{2} \mathrm{e}^{\eta T}+\phi_{2}(T) .
$$

Therefore, the efficient frontier (that is nonlinear and have parabolic shape) of the portfolio process is

$$
E\left(V^{*}(T)\right)=v_{0} \mathrm{e}^{\frac{1}{2} \eta T}+\frac{1}{\mathrm{i}} \sqrt{\sigma_{V^{*}(T)}^{2}-F(T)} .
$$

where,

$$
\mathrm{i}=\sqrt{-1} \text {. If } \sigma_{V^{*}(T)}^{2}=F(T) \geq 0,
$$

it implies that

$$
E\left(V^{*}(T)\right)=v_{0} \mathrm{e}^{\frac{1}{2} \eta T} .
$$

This shows that fund can be borrowed from the optimal wealth at time $t=0$ for $T$ year.

\section{Conclusion}

In this paper, we studied the optimal variational Merton portfolios with inflation protection strategy for a defined contribution Pension scheme. The present values of PPM's future contribution and sensitivity analysis of the present value of the contribution were established. The optimal variational Merton portfolio processes with inter-temporal hedging terms and inflation protection that offset any shocks to the stochastic salary of a PPM are obtained. Furthermore, expected values of PPM's terminal wealth and variance as well as the efficient frontier were obtained.

\section{REFERENCES}

[1] L. Cao and Z. F. Guo, "Optimal Variance Swaps Investments," IAENG International Journal of Applied Mathematics, Vol. 41, No. 4, 2011, 5 Pages.

[2] L. Cao and Z. F. Guo, "Delta hedging through Deltas from a Geometric Brownian Motion Process," Proceedings of International Conference on Applied Financial Economics, London, 30 June-2 July 2011.

[3] P. E. Davis, "Portfolio Regulation of Life Insurance Companies and Pension Funds," Oxford University Press, Oxford, 2000. http.//www.oecd.org/dataoecd/30/39/2401884.pdf

[4] A. J. G. Cairns, D. Blake and K. Dowd, "Stochastic Lifestyling: Optimal Dynamic Asset Allocation for Defined Contribution Pension Plans," Journal of Economic Dy- namics \& Control, Vol. 30, No. 6, 2006, pp. 843-877. http://dx.doi.org/10.1016/j.jedc.2005.03.009

[5] S. Brawne, A. M. Milevsky and T. S. Salisbury, "Asset Allocation and the Liquidity Premium for Illiquid Annuities," The Journal of Risk and Insurance, Vol. 70, No. 3, 2003, pp. 509-526.

http://dx.doi.org/10.1111/1539-6975.t01-1-00062

[6] G. Deelstra, M. Grasselli and P. Koehl, "Optimal design of the Guarantee for Defined Contribution Funds," 2002. http://www.amazon.com/Optimal-design-guarantee-defin ed-contribution/dp/B000RQY19I

[7] G. Deelstra, M. Grasselli and P. Koehl, "Optimal Investment Strategies in the Presence of a Minimum Guarantee," Insurance: Mathematics and Economics, Vol. 33, No. 1, 2003, pp. 189-207. http://dx.doi.org/10.1016/S0167-6687(03)00153-7

[8] G. Deelstra, M. Grasselli and P. Koehl, "Optimal Design of the Guarantee for Defined Contribution Funds," Journal of Economics Dynamics and Control, Vol. 28, No. 11, 2004, pp. 2239-226. http://dx.doi.org/10.1016/i.jedc.2003.10.003

[9] C. R. Nwozo and C. I. Nkeki, "Optimal Investment Strategy for a Defined Contributory Pension Plan in Nigeria Using Dynamic Optimization Technique," Studies in Mathematical Sciences, Vol. 2, No. 2, 2011, pp. 43-60.

[10] C. R. Nwozo and C. I. Nkeki, "Optimal Investment and Portfolio Strategies with Minimum Guarantee and Inflation Protection for a Defined Contribution Pension Scheme," Studies in Mathematical Sciences, Vol. 2, No. 2, 2011, pp. 78-89.

[11] C. R. Nwozo and C. I. Nkeki, "Optimal Portfolio and Strategic Consumption Planning in a Life-Cycle of a Pension Plan Member in A Defined Contributory Pension Scheme," IAENG International Journal of Applied Mathematics, Vol. 41, No. 4, 2011, p. 299.

[12] C. I. Nkeki, "On Optimal Portfolio Management of the Accumulation Phase of a Defined Contributory Pension Scheme," Ph.D. Thesis, University of Ibadan, Ibadan, 2011.

[13] C. I. Nkeki and C. R. Nwozo, "Variational Form of Classical Portfolio Strategy and Expected Wealth for a Defined Contributory Pension Scheme," Journal of Mathematical Finance, Vol. 2, No. 1, 2012, 132-139. http://dx.doi.org/10.4236/jmf.2012.21015

[14] C. I. Nkeki and C. R. Nwozo, "Optimal Investment under Inflation Protection and Optimal Portfolios with Stochastic Cash Flows Strategy," To appear in IAENG Journal of Applied Mathematics, Vol. 43, No. 2, 2013, p. 54. 